05,06

\title{
Электронная структура и несобственная электрическая поляризация ортоферрита самария
}

\author{
(C) В.В. Тригук ${ }^{1}$, И.И. Макоед ${ }^{1, \uparrow}$, А.Ф. Ревинский ${ }^{2}$ \\ ${ }^{1}$ Брестский государственный университет им. А.С. Пушкина, \\ Брест, Беларусь \\ ${ }^{2}$ Белостокский технический университет, \\ Белосток, Польша \\ ฯ E-mail: igmak2010@yandex.ru
}

(Поступила в Редакцию 28 апреля 2016 г.)

\begin{abstract}
Из первых принципов методом функционала плотности в рамках приближения LSDA $+U$ рассчитаны зонная структура, распределения электронной и спиновой плотностей ортоферрита самария с учетом коллинеарного антиферромагнитного упорядочения магнитных моментов катионов железа и самария. На основании результатов теоретико-группового анализа рассмотрена возможность индуцирования сегнетоэлектрического состояния при температурах, меньших точки антиферромагнитного упорядочения магнитной подрешетки, образованной катионами самария. В области высоких температур возникновение областей со спонтанной электрической поляризацией возможно при наличии дополнительных факторов, понижающих симметрию кристалла.
\end{abstract}

Работа выполнена в рамках Государственной программы научных исследований на 2016-2020 гг. „Физическое материаловедение, новые материалы и технологии“.

\section{1. Введение}

Феррит самария $\mathrm{SmFeO}_{3}$ (SFO) относится к семейству редкоземельных ортоферритов с перовскитоподобной кристаллической структурой (пр.гр. $D_{2 h}^{16}-P b n m$ ) [1]. В последнее время данный материал привлекает внимание исследователей в связи с обнаружением в нем несобственной сегнетоэлектрической поляризации $\left(T_{C}=670 \mathrm{~K}\right): P_{s}=100 \mu \mathrm{C} / \mathrm{m}^{2}$ [2]. Ранее было установлено $[3,4]$, что в $\mathrm{SFO}$ реализуется антиферромагнитное (АФМ) упорядочение $G$-типа спинов катионов железа $\mathrm{Fe}^{3+}$ при температурах, меньших $T_{N 1}=670 \mathrm{~K}$. В работах $[2,5,6]$ описан обнаруженный в образцах SFO слабый ферромагнетизм, обусловленный нарушением коллинеарности (скосом) спиновых моментов подрешеток, образованных магнитоактивными катионами $\mathrm{Fe}^{3+}$ $\left(d\right.$-подрешетка) и $\mathrm{Sm}^{3+}(f$-подрешетка). При низких температурах $T<T_{N 2}=10 \mathrm{~K}$ спины катионов самария также образуют АФМ-порядок [3], как это показано на рис. 1. Совпадение точек Кюри и Нееля $\left(T_{C}=T_{N 1}\right)$ дает основание отнести данное соединение к мультиферроикам второго рода, в которых сегнетоэлектрическая (СЭ) фаза индуцируется магнитоэлектрическим (МЭ) взаимодействием [7].

Сложный характер температурно-обусловленной спиновой динамики магнитоактивных катионов приводит к наличию в SFO ряда фазовых превращений. В частности, при температуре $T_{\mathrm{SR}}=480 \mathrm{~K}$ происходит спин-переориентационный переход, в результате которого изменяется магнитная симметрия $d$-подрешетки: $\Gamma_{2}\left(F_{x}^{d} C_{y}^{d} G_{z}^{d}\right) \rightarrow \Gamma_{4}\left(G_{x}^{d} A_{y}^{d} F_{z}^{d}\right) \quad[2,5,6]$. При температуре компенсации $\left(T^{*}=5 \mathrm{~K}\right)$ наблюдается изменение знака намагниченности SFO на противоположный $[2,5]$. Это открывает перспективы для возможного использования ортоферрита самария в качестве материала для создания высокоскоростных переключателей [5] и многопараметрических устройств спинтроники [8].

Теория магнитной симметрии $d$ - и $f$-подсистем в ортоферритах хорошо разработана. Пространственная группа Pbnm является центрально-симметричной (включает операцию инверсии). Магнитная элементарная ячейка совпадает с кристаллической. Относительно природы несобственной электрической поляризации и магнитоэлектрических взаимодействий в $d$ - и $f$-подрешетках SFO в литературе приведены противоречивые данные $[2,9]$. В связи с этим представляет интерес проведение $a b$ initio расчетов распределения электронной и спиновой плотности, а также теоретико-групповой анализ возможных упорядоченных магнитных и зарядовых состояний в SFO с целью выяснения причин сосуществования СЭ- и АФМ-фаз.

Целью настоящей работы является теоретическое исследование условий совместимости антиферромагнитного упорядочения и сегнетоэлектрической поляризации в $\mathrm{SmFeO}_{3}$ на основании данных $a b$ initio расчетов зонной структуры, распределения спиновой плотности и результатов теоретико-группового анализа.

\section{2. Расчет зонной структуры}

Для выполнения теоретических расчетов зонной структуры использовался метод функционала электронной плотности, реализованный в программном комплекce ABINIT [10]. Соединения с частично заполненными внутренними $d$ - и $f$-оболочками являются веществами с 


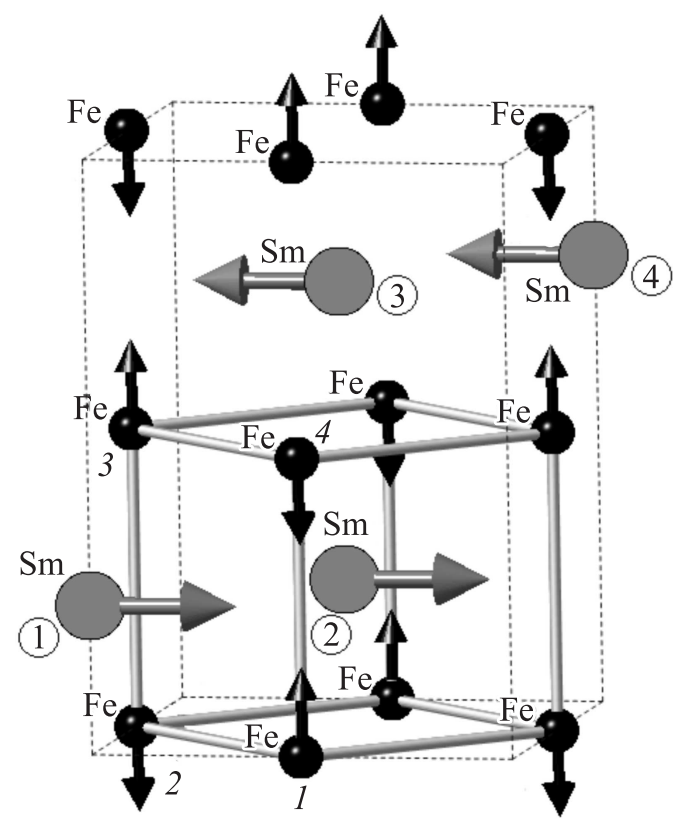

Pис. 1. Магнитная структура $\mathrm{SmFeO}_{3}$. Цифры соответствуют атомам Fe, цифры в кружках - атомам Sm.

сильнокоррелированной электронной подсистемой. Поэтому при расчетах для обменно-корреляционного взаимодействия было использовано приближение LSDA $+U$ (LSDA - local spin density approximation) в гамильтониане Кона-Шема [11]. Расчеты проводились для однородных коллинеарных АФМ-упорядочений $G$-типа ( $d$-подрешетка) и $C$-типа ( $f$-подрешетка).

Как было показано в работе [6], теоретические значения параметров кристаллической решетки ортоферритов слабо зависят от типа магнитной симметрии. Оптимизацию кристаллической структуры проводили, интегрируя в зоне Бриллюэна по схеме $5 \times 5 \times 5$ до достижения величин сил Гельмана-Феймана, равных $10^{-6} \mathrm{Ha} / \mathrm{Bohr}$. Максимальная кинетическая энергия плоских волн задавалась равной 25 На. В качестве валентных учитывалось шестнадцать электронов для $\mathrm{Sm}\left(4 f^{5} 5 s^{2} 5 p^{6} 5 d^{1} s^{2}\right)$, восемь для $\mathrm{Fe}\left(3 d^{6} 4 s^{2}\right)$ и шесть для $\mathrm{O}\left(2 s^{2} 2 p^{4}\right)$. Параметры корреляционного взаимодействия задавались равными

Равновесные значения координат позиций Уайкова и параметров решетки $\mathrm{SmFeO}_{3}$

\begin{tabular}{|c|c|c|c|c|c|c|}
\hline Параметр & \multicolumn{3}{|c|}{$\begin{array}{c}\text { Данные настоящей } \\
\text { работы }\end{array}$} & \multicolumn{3}{|c|}{ Данные [14] } \\
\hline $\mathrm{Fe}^{3+}(4 b)$ & 0.5 & 0 & 0 & 0.5 & 0 & 0 \\
\hline $\mathrm{Sm}^{3+}(4 c)$ & 0.4985 & 0.5038 & 0.25 & 0.5134 & 0.5572 & 0.25 \\
\hline $\mathrm{O}_{1}^{2-}(4 c)$ & 0.5014 & 1.0044 & 0.25 & 0.3958 & 0.9894 & 0.25 \\
\hline $\mathrm{O}_{2}^{2-}(8 d)$ & 0.2247 & 0.7168 & 0.9999 & 0.2862 & 0.6966 & 0.9613 \\
\hline$a, \AA$ & & 5.3979 & & & 5.4026 & \\
\hline$b, \AA$ & & 5.6480 & & & 5.6001 & \\
\hline$c, \AA$ & & 7.6574 & & & 7.7129 & \\
\hline
\end{tabular}

$U_{\mathrm{eff}}=4 \mathrm{eV}$ для $3 d$-электронов железа и $U_{\mathrm{eff}}=6 \mathrm{eV}$ для $4 f$ - и $5 d$-электронов самария. Как показали результаты более ранних расчетов, при указанных значениях $U_{\text {eff }}$ происходит относительная стабилизация зонной структуры $[12,13]$.

В таблице представлены вычисленные значения параметров решетки SFO в сравнении с известными экспериментальными данными. Для оптимизации параметров кристаллической решетки была использована коллинеарная магнитная структура, изображенная на рис. 1. Результаты расчетов показывают, что минимальному значению полной энергии соответствуют следующие магнитные моменты: $\mu\left(\mathrm{Fe}^{3+}\right)=(0,0,3.68) \mu_{\mathrm{B}}$ и $\mu\left(\mathrm{Sm}^{3+}\right)=(0,5.40,0) \mu_{\mathrm{B}}$. Величина магнитного момента, рассчитанная для катиона $\mathrm{Fe}^{3+}$, близка к значению, полученному в работе [15]. Распределение разностной спиновой плотности $\Delta \rho=\rho \uparrow-\rho \downarrow$ указывает на тенденцию к противоположной ориентации магнитных моментов катионов $d$ - и $f$-подсистем.

Рассчитанные зонные структуры SFO для различных направлений спинов валентных электронов (spin up и spin down) представлены на рис. 2,3. Как следует из результатов расчетов полной электронной плотности, приведенных на рис. 4, $a$, ортоферрит самария является полупроводником с шириной запрещенной зоны $E_{g}=2.0 \mathrm{eV}$. Полученная величина хорошо согласуется с расчетными данными работы [16]. Зона проводимости

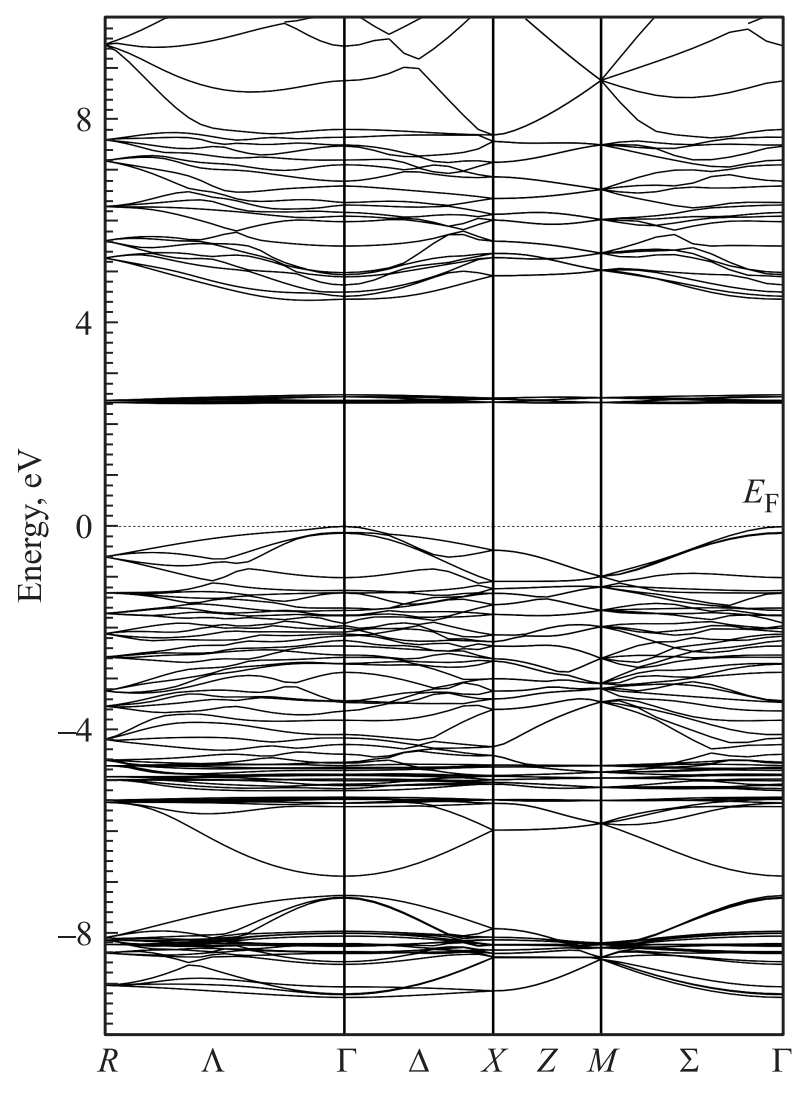

Pис. 2. Зонная структура $\mathrm{SmFeO}_{3}$ для состояний spin up. 


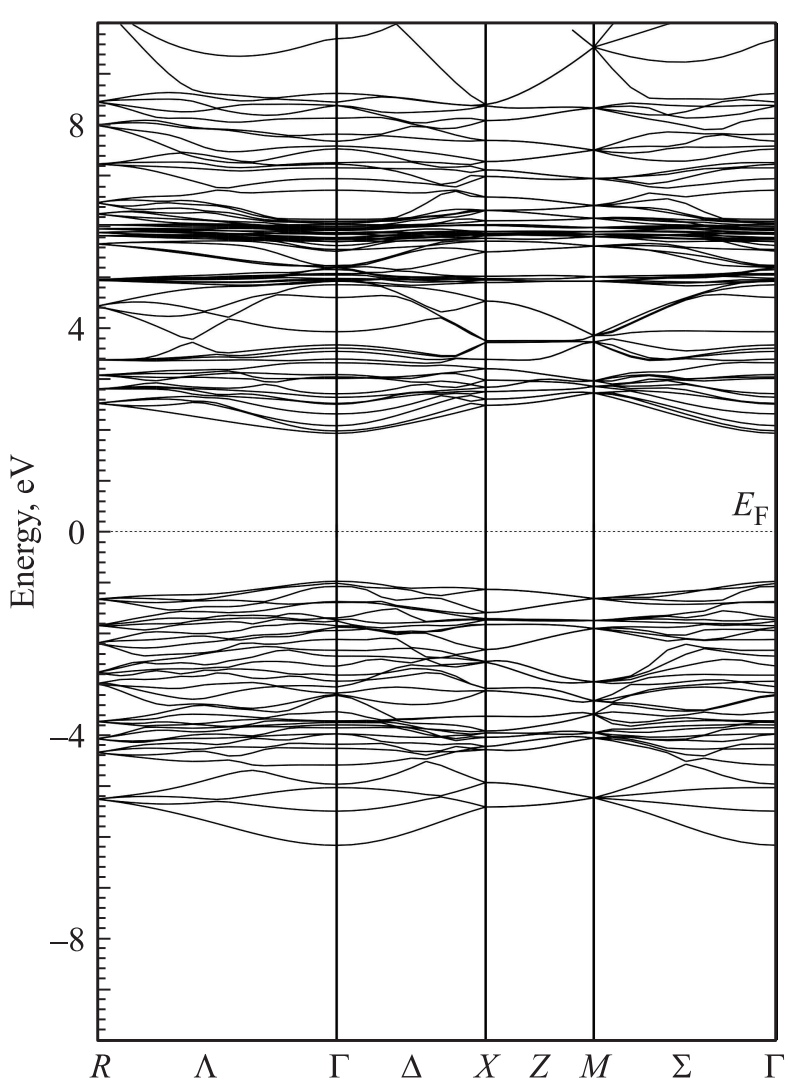

Рис. 3. Зонная структура $\mathrm{SmFeO}_{3}$ для состояний spin down.
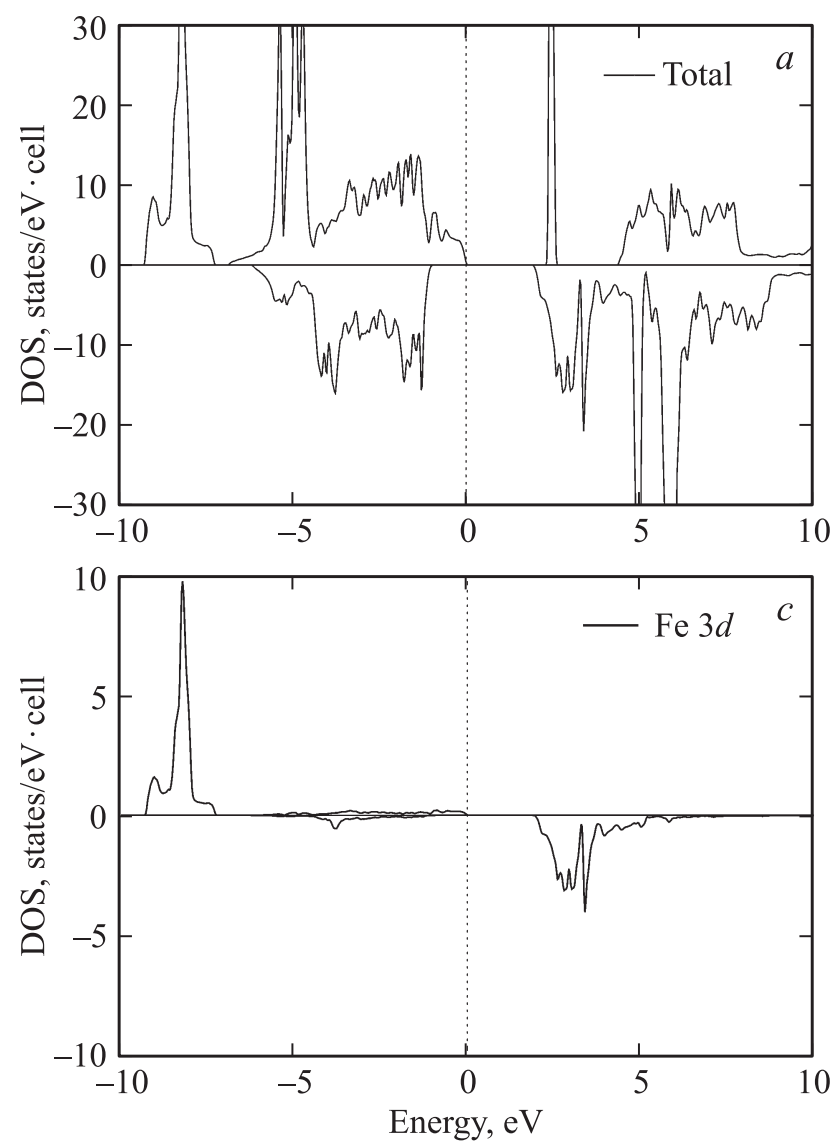

SFO сформирована в основном spin up состояниями и является своеобразным „фильтром“ для электронов с определенной ориентацией спинового магнитного момента. Анализ распределения парциальных плотностей электронных состояний свидетельствует о том, что данная особенность в формировании зоны проводимости в значительной мере обусловлена $3 d$-состояниями железа, показанными на рис. $4, c$. Энергетические уровни сильнокоррелированных $3 d$-состояний железа и $4 f$-состояний самария, представленных на рис. $4, b$, локализованы в значительной степени в валентной зоне с положительным (spin up) направлением спина.

Величина вклада $3 d$-электронов катионов железа в полную плотность состояний вблизи уровня Ферми составляет примерно 20\%. Дно зоны проводимости сформировано $3 d$-состояниями катионов железа с отрицательным (spin down) направлением спина и $4 f$-состояниями катионов самария с положительным (spin up) направлением спина. Потолок валентной зоны, как видно на рис. $4, d$, представлен, в основном, $2 s$ и $2 p$-состояниями электронов кислорода. Полученная картина распределения электронных состояний $\mathrm{SFO}$ является типичной для ортоферритов $\mathrm{GdFeO}_{3}, \mathrm{TbFeO}_{3}$, $\mathrm{EuFeO}_{3}$, результаты расчета зонных структур которых приведены в работе [17].

Слабый ферромагнетизм SFO обусловлен скосом магнитных подрешеток катионов $\mathrm{Fe}^{3+}$ и $\mathrm{Sm}^{3+}$. Как видно
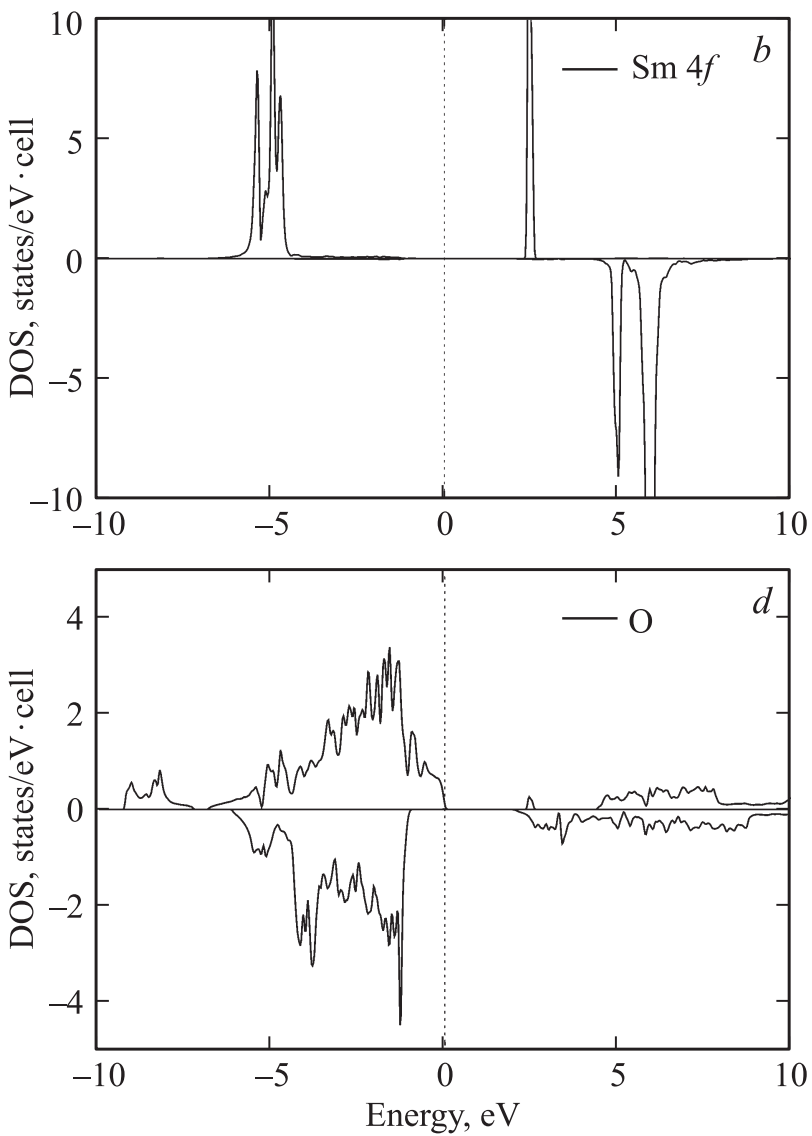

Рис. 4. Полная $(a)$ и парциальные плотности электронных состояний $\mathrm{Sm} 4 f(b), \mathrm{Fe} 3 d(c)$ и $\mathrm{O}(d)$ в $\mathrm{SmFeO}_{3}$. 


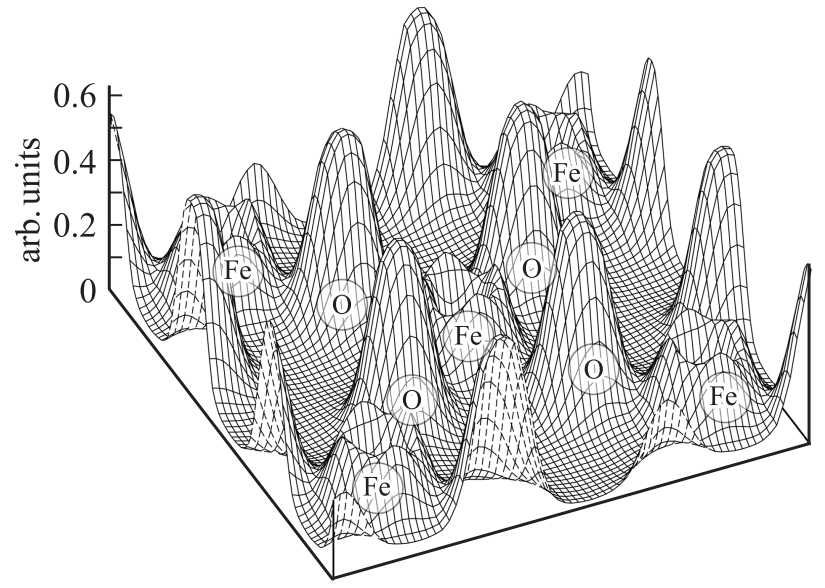

Рис. 5. Распределение электронной плотности в $d$-плоскости $\mathrm{SmFeO}_{3}$.

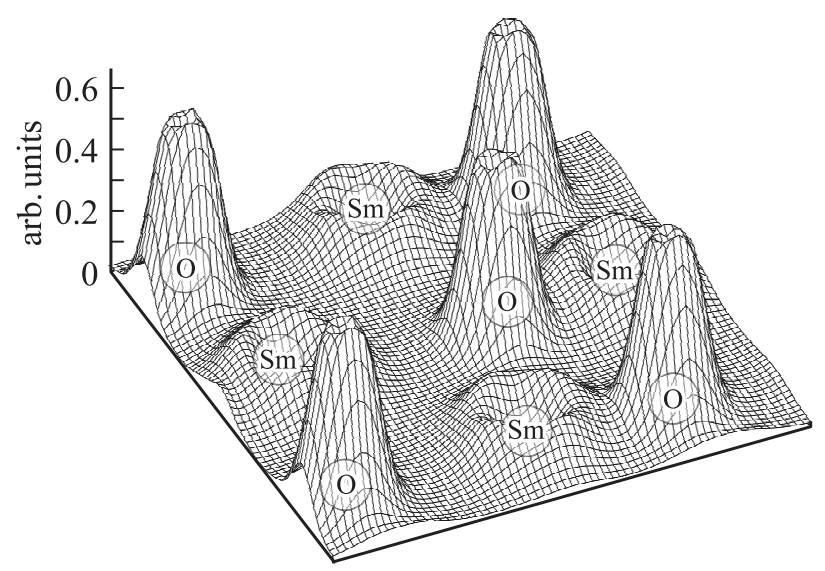

Рис. 6. Распределение электронной плотности в $f$-плоскости $\mathrm{SmFeO}_{3}$.

из рис. 5 и 6, между катионами железа и самария наблюдается высокая плотность валентных электронов лигандов $\mathrm{O}^{2-}$, которые занимают положения Уайкова $4 c$ и $8 d$. Полученные результаты свидетельствуют о преобладающей роли косвенных обменных взаимодействий $\mathrm{Fe}^{3+}-\mathrm{O}^{2-}-\mathrm{Fe}^{3+}$ и $\mathrm{Sm}^{3+}-\mathrm{O}^{2-}-\mathrm{Sm}^{3+}$ над прямыми взаимодействиями $\mathrm{Fe}^{3+}-\mathrm{Fe}^{3+}, \mathrm{Sm}^{3+}-\mathrm{Sm}^{3+}$, $\mathrm{Fe}^{3+}-\mathrm{Sm}^{3+}$.

\section{3. Магнитоэлектрическое взаимодействие в SFO}

Необходимым условием сосуществования магнитного упорядочения и СЭ-поляризации в кристалле является нарушение пространственной и временно́й инверсии [7]. В ортоферрите самария $d$-подрешетка является центрально-симметричной, а следовательно, неактивной в плане МЭ-взаимодействия и сосуществования АФМ- и СЭ-упорядочений. Центрально-асимметричная $f$-подрешетка допускает наличие сегнетоэлектрической поляризации в отсутствие внешних электрического и магнитного полей. Представленные на рис. 6 данные подтверждают асимметричный характер распределения электронной плотности SFO в $f$-плоскости и указывают на возможность установления упорядоченного состояния со спонтанной электрической поляризацией $\mathbf{P}_{s}$.

Теоретические основы магнитных структур ортоферритов в настоящее время достаточно хорошо разработаны $[3,18,19]$. Слабый ферромагнетизм SFO обусловлен нарушением коллинеарности спиновых магнитных моментов $\mathbf{S}_{i}^{d}$ катионов $\mathrm{Fe}^{3+}$ и $\mathbf{S}_{i}^{f}$ катионов $\mathrm{Sm}^{3+}[5,18]$. Согласно результатам нейтронографических исследований, изложенным в работах $[2,5,6]$, магнитная структура $d$-подрешетки $\mathrm{SFO}$ при $T_{\mathrm{SR}}<T<T_{N 1}$ характеризуется симметрией $\Gamma_{4}\left(G_{x}^{d} A_{y}^{d} F_{z}^{d}\right)$, а при $T<T_{\mathrm{SR}}$ симметрией $\Gamma_{2}\left(F_{x}^{d} C_{y}^{d} G_{z}^{d}\right)$ (рис. 7,a,b). При низких температурах $T_{N 2}<10 \mathrm{~K}$ в кристалле формируется дополнительный вклад, обусловленный слабым прямым обменным взаимодействием $\mathrm{Sm}^{3+}-\mathrm{Sm}^{3+}$. В результате в $\mathrm{SFO}$ устанавливается АФМ-тип магнитного упорядочения в обеих магнитных подрешетках.

Неколлинеарные векторы $\mathbf{S}_{i}^{d}$ для симметрии $\Gamma_{2}\left(F_{x}^{d} C_{y}^{d} G_{z}^{d}\right)$ в общем случае имеют следующие компоненты: $\mathbf{S}_{1}^{d}=(-u, v, w), \quad \mathbf{S}_{2}^{d}=(-u, v,-w), \quad \mathbf{S}_{3}^{d}=$ $=(-u,-v,-w), \quad \mathbf{S}_{4}^{d}=(-u,-v, w)$, где $u, v, w-$ декартовы компоненты в единицах $\mu_{\mathrm{B}}$. Такая структура соответствует магнитному упорядочению $G$-типа вдоль оси $z$. Намагниченность обусловлена нарушением коллинеарности $(u \neq 0, \quad v \neq 0)$ и равна $M^{d}=-4 u$ $\left(\mu_{\mathrm{B}} /\right.$ cell $)$. Направление вектора $\mathbf{M}^{d}=(-4 u, 0,0)$ противоположно направлению оси $x$. Магнитная структура ионов $\mathrm{Sm}^{3+}(f$-подсистема $) \Gamma_{2}\left(F_{x}^{f} C_{y}^{f}\right)$ соответствует антиферромагнитному упорядочению $C$-типа вдоль оси $y$ и может быть задана вектором $\mathbf{M}^{f}=\left(2\left(p-p^{\prime}\right), 0,0\right) \quad \mathrm{c}$ компонентами $\mathbf{S}_{1}^{f}=(p, q, 0)$, $\mathbf{S}_{2}^{f}=(p,-q, 0), \mathbf{S}_{3}^{f}=(p,-q, 0), \mathbf{S}_{4}^{f}=(p, q, 0)$. Нарушение коллинеарности $(p \neq 0)$ обусловливает намагниченность $M^{f}=4 p\left(\mu_{\mathrm{B}} /\right.$ cell $)$, направленную противоположно вектору $\mathbf{M}^{d}$. Результирующая намагниченность определяется выражением $\mathbf{M}=\mathbf{M}^{d}-\mathbf{M}^{f}$, которое позволяет объяснить в рамках двухподрешеточной модели экспериментально наблюдаемые особенности температурной зависимости величины удельной намагниченности. Согласно законам симметрии представление $\Gamma_{2}\left(F_{x}^{d} C_{y}^{d} G_{z}^{d}\right)$, для $d$-подрешетки совместимо с изображенным на рис. 7, $c$ представлением $\Gamma_{2}\left(F_{x}^{f} C_{y}^{f}\right) \quad f$-подрешетки [3]. Тогда полученному представлению $\Gamma_{2}\left(F_{x}^{d} C_{y}^{d} G_{z}^{d} ; F_{x}^{f} C_{y}^{f}\right)$ соответствует точечная магнитная группа $\mathrm{mm}^{\prime} \mathrm{m}^{\prime}[9,18]$, которая является неполярной; следовательно, наблюдаемая экспериментально спонтанная СЭ-поляризация SFO не может быть индуцирована за счет МЭ-взаимодействия.
МЭ-активную спиновую конфигурацию $\Gamma_{25}\left(F_{x}^{f} C_{y}^{f} ; G_{x}^{f} A_{y}^{f}\right)$ для $f$-подрешетки SFO, приведенную на рис. $7, d$, можно получить при помощи комбинации представлений $\Gamma_{2}\left(F_{x}^{f} C_{y}^{f}\right)$ и $\Gamma_{5}\left(G_{x}^{f} A_{y}^{f}\right)$. Спиновой конфи- 

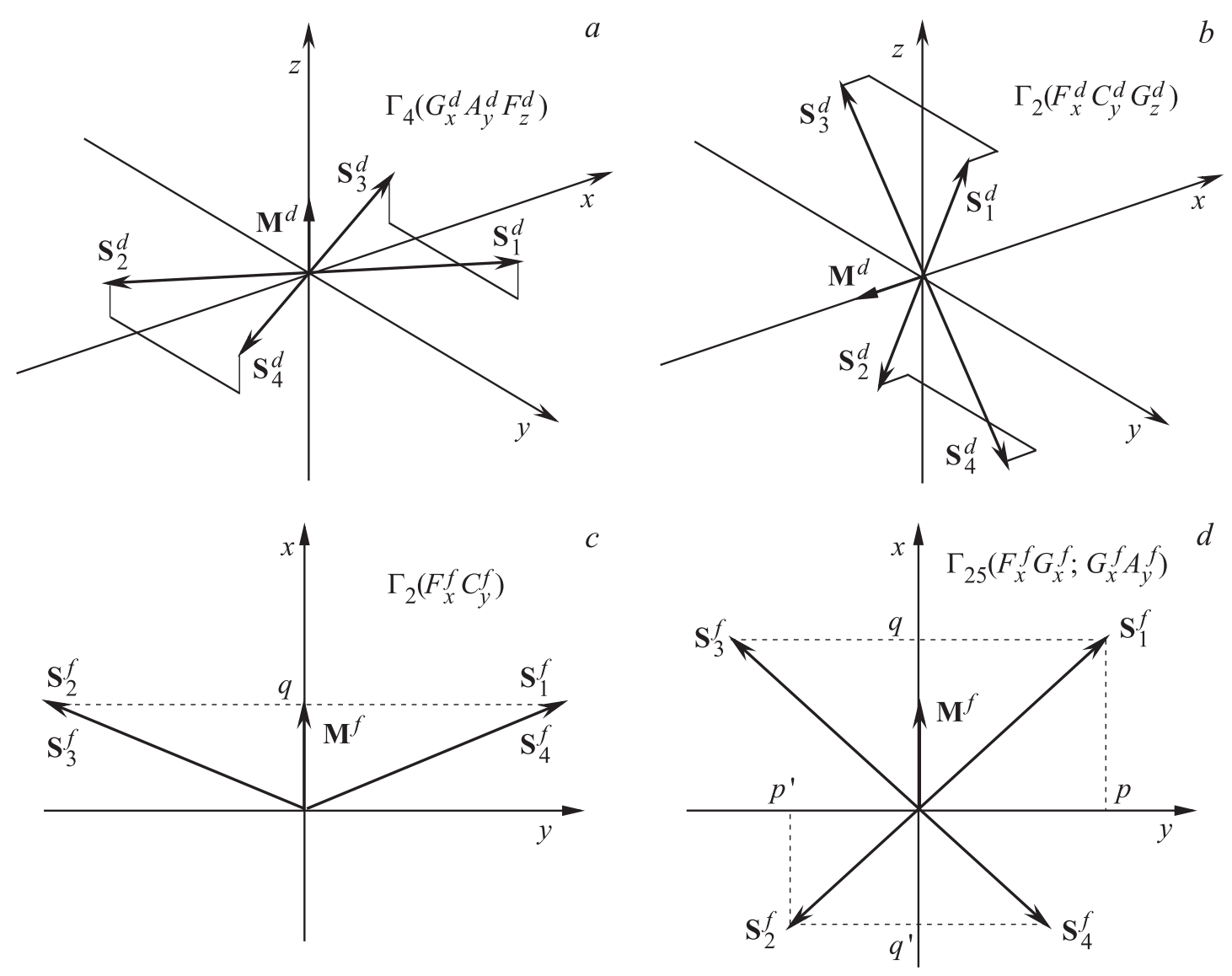

Рис. 7. Магнитные конфигурации $d$ - $(a, b)$ и $f$-подрешеток $(c, d) \mathrm{SmFeO}_{3}$.

гурации $\Gamma_{25}\left(F_{x}^{d} C_{y}^{d} G_{z}^{d} ; F_{x}^{f} C_{y}^{f} ; G_{x}^{f} A_{y}^{f}\right)$ при низких $\left(T<T_{N 2}\right)$ температурах соответствует магнитная точечная группа $m m^{\prime} 2$, которая является полярной [18]. Компоненты магнитных моментов $\mathbf{S}_{i}^{f}$ ионов $\mathrm{Sm}^{3+}$ для полученного представления $\Gamma_{25}$ имеют вид $\mathbf{S}_{1}^{f}=(p, q, 0), \quad \mathbf{S}_{2}^{f}=$ $=\left(-p^{\prime}, q, 0\right), \quad \mathbf{S}_{3}^{f}=(p,-q, 0), \quad \mathbf{S}_{4}^{f}=\left(-p^{\prime},-q^{\prime}, 0\right)$. Peзультирующий магнитный момент подрешетки катионов самария равен $\mathbf{M}^{f}=\left(2\left(p-p^{\prime}\right), 0,0\right)$. При этом намагниченности $d$ - и $f$-подрешеток противоположны и направлены вдоль оси $x$.

Данная модель позволяет объяснить наличие температуры компенсации, при которой $\mathbf{M}^{f}=-\mathbf{M}^{d}$, а также допускает возможность индуцирования в результате МЭ-взаимодействия, т.е. без влияния внешних электрического или магнитного полей, спонтанной СЭ-поляризации $\mathbf{P}_{s}=\left(0, p_{y}, 0\right)$, экспериментально наблюдаемой при $T<T_{N 2}[2]$. С ростом температуры при $T>T_{N 2}$ АФМ-упорядочение магнитных моментов катионов $\mathrm{Sm}^{3+}$ разрушается, и $f$-подрешетка переходит в парамагнитное состояние. При этом $d$-подрешетка продолжает сохранять две магнитные конфигурации $\Gamma_{2}\left(F_{x}^{d} C_{y}^{d} G_{z}^{d}\right)$ при $T<T_{\mathrm{SR}}$ и $\Gamma_{4}\left(G_{x}^{d} A_{y}^{d} F_{z}^{d}\right)$ при $T_{\mathrm{SR}}<T<T_{N 2}$, оставаясь центрально-симметричной. В области высоких температур спонтанная СЭ-поляризация запрещена законами симметрии, и для объяснения ее наличия следует признать возможным существование в SFO дополнительных факторов, понижающих симметрию кристалла. В качестве последних могут выступать как магнитоупругие взаимодействия, так и локальные понижения симметрии за счет возникновения фрустрированной магнитной структуры.

\section{4. Заключение}

Результаты $a b$ initio расчетов зонной структуры показывают, что в основном состоянии ортоферрит самария является полупроводником с шириной запрещенной зоны $2 \mathrm{eV}$. Определяющая роль в формировании энергетических полос вблизи уровня Ферми принадлежит сильнокоррелированным $3 d$-состояниям электронов катионов железа и $f$-состояниям электронов катионов самария. Топография карт распределения электронной плотности указывает на наличие выраженной ее асимметрии в $f$-подрешетке и служит основанием для связи несобственной спонтанной СЭ-поляризации в области низких температур с магнитоэлектрическим взаимодействием. При этом спиновая конфигурация должна соответствовать представлению $\Gamma_{25}\left(F_{x}^{d} C_{y}^{d} G_{z}^{d} ; F_{x}^{f} C_{y}^{f} ; G_{x}^{f} A_{y}^{f}\right)$ магнитной симметрии $d$ - и $f$-подрешеток. 


\section{Список литературы}

[1] А.К. Звездин, В.М. Матвеев, А.А. Мухин, А.И. Попов. Редкоземельные ионы в магнитоупорядоченных кристаллах. Наука, М. (1985). 296 с.

[2] J.-H. Lee, Y.K. Jeong, J.H. Park, M.-A. Oak, H.M. Jang, J.Y. Son, J.F. Scott. Phys. Rev. Lett. 107, 117201 (2011).

[3] R.L. White. J. Appl. Phys. 40, 3, 1061 (1969).

[4] E.N. Maslen, V.A. Streltsov, N. Ishizawa. Acta Cryst. B 52, 406 (1996).

[5] Y.K. Jeong, J.-H. Lee, S.-J. Ahn, H.M. Jang. Solid State Commun. 152, 1112 (2012).

[6] L.G. Marshall, J.-G. Cheng, J.-S. Zhou, J.B. Goodenough, J.-Q. Yan, D.G. Mandrus. Phys. Rev. B 86, 064417 (2012).

[7] А.К. Звездин, А.П. Пятаков. УФН 182, 6, 594 (2012).

[8] X. Wang, X. Cheng, S. Gao, J. Song, K. Ruan, X. Li. J. Magn. Magn. Mater. 399, 170 (2016).

[9] R.D. Johnson, N. Terada, P.G. Radaelli. Phys. Rev. Lett. 108, 219701 (2012)

[10] X. Gonze, G.M. Rignanese, M. Verstraete, J.M. Beuken, Y. Pouillon, R. Caracas, F. Jollet, M. Torrent, G. Zerah, M. Mikami, P. Ghosez, M. Veithen, J.Y. Raty, V. Olevano, F. Bruneval, L. Reining, R. Godby, G. Onida, D.R. Hamann, D.C. Allan. Comp. Phys. Commun. 220, 558 (2005).

[11] S.L. Dudarev, L.-M. Peng, S.Y. Savrasov, J.-M. Zuo. Phys. Rev. B 61, 2506 (2000)

[12] А.Ф. Ревинский, В.В. Тригук, И.И. Макоед. ФТТ 56, 9, 1739 (2014).

[13] L. Chen, T. Li, S. Cao, S. Yuan, F. Hong, J. Zhang. J. Appl. Phys. 111, 103905 (2012).

[14] N.N. Li, H. Li, R.-L. Tang, D.-D. Han, Y.-S. Zhao, W. Gao, P.-W. Zhu, X. Wang. Chin. Phys. 23, 046105 (2014).

[15] В.С. Жандун, В.И. Зиненко. ФТТ 57, 5, 970 (2015).

[16] N. Singh, J.Yu. Rhee, S. Auluck. J. Korean Phys. Soc. 53, 2, 806 (2008).

[17] D. Mekam, S. Kacimi, M. Djermouni, M. Azzouz, A. Zaoui. Results Phys. 2, 156 (2012).

[18] T. Yamaguchi, K. Tsushima. Phys. Rev. B 8, 11, 5187 (1973).

[19] А.К. Звездин, А.А. Мухин. Письма в ЖЭТФ 88, 8, 581 (2008). 